atory should be followed. Since some anaerobes are fairly tolerant to oxygen contact, many laboratories will have some degree of success in isolating non-fastidious anaerobes. But they may be overlooking many more demanding anaerobes unless they use the best technique of isolation.

Susceptibility testing of anaerobes showed the following results : 1) aminoglycosides, colistin and polymyxin-B are inactive against all anaerobes; 2) lincomycin, clinda- mycin and chloramphenicol are active against all anaerobes; 3) cephalosporins, penicillin $G$ and its semisynthetic derivatives are active against gram-positive cocci and rods but inactive against gram-negative rods.

Although clindamycin is the drug of choice for all anaerobic infections, an appreciable number of recent isolates of $B$. fragilis have been shown to be resistant to clindamycin.

\title{
(3) Side Effects of Antibacterial Chemotherapeutics
}

\author{
Hiroshi Okuвo, M D
}

The First Department of Internal Medicine, Kansai Medical University

In the main, chemotherapeutic side effects can be divided into two groups according to their mechanisms: the toxic and the allergic. But in dealing with the obscure border between these two groups, the concept of hypersensitivity, which includes some allergic and allergic-like reactions, is of practical use.

I. With the cooperation of the leading hospitals all over the country, we were able to make serial surveys on drug-hypersensivity cases, among which $38.3 \%$ (530 cases) from 1967 to 1970 and 44\% (609 cases) from 1971 to 1975 had been induced by antibacterial chemotherapeutics (Table 1).

Table 1. Number of hypersensitives cases (Chemotherapeutics) (1971-1975).

\begin{tabular}{|c|c|c|c|c|c|c|c|}
\hline \multicolumn{2}{|c|}{ Disorder Type: } & Skin & Blood & Liver & Kidney & Fever & Shock \\
\hline Drugs & Net & & & & & & \\
\hline Antibiotics & 471 & 379 & 127 & 103 & 31 & 105 & 11 \\
\hline Sulfonamides & 78 & 72 & 4 & 13 & 4 & 22 & 0 \\
\hline Anti-Tbc. Ag. & 37 & 18 & 6 & 12 & 3 & 7 & 0 \\
\hline Others & 10 & 8 & 4 & 2 & 2 & 0 & 1 \\
\hline Combined & 13 & 9 & 3 & 8 & 2 & 6 & 0 \\
\hline Total & 609 & 486 & 144 & 138 & 42 & 140 & 12 \\
\hline$\frac{\text { Cases of Chemoth. }}{\text { Cases of All Drugs }} \times 100 \%$ & 44.0 & 48.2 & 54.8 & 40.2 & 40.8 & 44.4 & 33.3 \\
\hline
\end{tabular}

The most frequently observed sensitivity phenomena are skin lesions, followed by blood disorders, fever, and liver damage.
Each drug group tends to favor a certain type of disorder, e.g. chloramphenicol (CP) might affect the blood while macrolides (MACs) and tetracyclines (TGs) might affect the liver (Fig. 1).

\begin{tabular}{|c|c|c|c|c|c|c|}
\hline \multicolumn{2}{|c|}{ Disorder of } & \multirow{2}{*}{ Blood } & \multirow{2}{*}{ Liver } & \multirow{2}{*}{$\begin{array}{c}\text { Kid- }^{-} \\
\text {ney }\end{array}$} & \multirow{2}{*}{ Shock } & \multirow{2}{*}{ Net } \\
\hline Drug & & & & & & \\
\hline PCs & & ص & ㅁ & ㄴ & ㅁ & 117 \\
\hline CEPs & & ๒ & [ & ら & 几 & 64 \\
\hline TCs & & ח & $\square$ & & & 20 \\
\hline $\mathrm{CP}$ & $\square$ & $\Omega_{3}$ & 凸 & ๒ & a & 90 \\
\hline AMGs & 17 & & ঢ & ص & 口 & 37 \\
\hline MACs & & לי & $\square$ & & 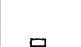 & 33 \\
\hline SAs & & 口 & ᄃ & 口 & & 70 \\
\hline
\end{tabular}

Fig. 1. Percentage of disorder types among hypersensitivity cases.

1) Anaphylactoid shock: Incidence is recently diminishing. The main responsible 
drugs are the penicillins (PGs), cephalosporins (CEPs), and CP.

2) Blood disorders: Granulocytopenia $(\mathrm{G})$ is the most frequently observed disorder, followed by aplastic anemia (A). CP tends to induce A, while the PCs, GEPs, and PAS tend to cause G. Thiamphenicol, a derivative of CP, seems to prefer pure erythroid hypoplasia. Pure thrombocytopenia is most frequently induced by the sulfonamides (SAs).

3) Liver damages: Recently reduced is the number of cases induced by the SAs, their place having been taken by the GEPs and rifampicin (RFP). In cases induced by aminoglycosides (AMGs), the seral ALP levels are rarely elevated as they are in those by the MACs and RFP.

4) Skin lesions: Besides being the most frequent signs of drug-hypersensitivity, they are important diagnostic clues. The fixed eruptions, the only type characteristic of drug-induced skin lesions, are mostly caused by the SAs.

5) Eosinophilia: For the 79 accumulated cases of eosinophilia (eosinophil leucocytes in the peripheral blood $>10 \%$ ) the most responsible drugs were ampicillin, AMGs, SAs, and PAS. In some of these cases, eosinophilia was accompanied by neutropenia.

6) Drug fever: Although skin eruptions or other lesions often accompany fever, drug-hypersensitivity sometimes yields fever without any other manifestations, in which case, the drug fever can easily be mistaken for a relapse of the original infection.

II. Diathetic considerations with regard to the pathogenesis of drug side effects seem to be very important, as, generally speaking, the side effects, especially in hypersensitivity cases, are of very low incidence, and usually dose-independent. Our studies on the age and sex distributions of these cases in comparison with those of the whole population, revealed that females in the third decade and males over 60 years of age are significantly disposed to drughypersensitivity. On the other hand, some are found to be sensitive to various drugs of quite different chemical structure. Pre- existing renal or hepatic dysfunctions, too, may build up a diathesis, by altered degraduation or excretion of the administered drug. Combined dosage of other drugs can also exert some influences on the incidence of untoward reactions to chemotherapeutics. Fig. 2 charts the probable diathetic constituents.

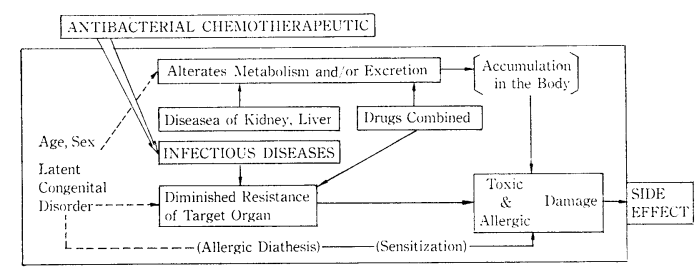

Fig. 2. Diathetic factors participating in the pathogenesis of disorders caused by antibacterial chemotherapeutics.

On this problem, we carried out some clinical and experimental studies:

1) Studies on GP-induced blood disorders: The ${ }^{3} \mathrm{HTdR}$ uptake by the bone marrow of rats treated with CP at dosages as high as 0.5 or $1.0 \mathrm{~g} / \mathrm{kg}$ showed no remarkable depression compared with the controls; but this uptake was depressed when the rats had been pre-treated with cyclophosphamide (CPM) $10 \mathrm{mg} / \mathrm{kg}$ seven days before CP, the nucleated cell count decreasing remarkably compared with those treated with CPM alone. On the other hand, rabbits treated with nitrogen mustard n-oxide (NMO) showed more remarkable and persistent neutropenia when NMO was administered combined with GP.

2) Studies on the influence of liver damages: Patients with liver diseases often maintain higher blood levels of antibiotics (josamycin, cephazolin, etc.) than normal persons, then approaching the latter in concomitance with the recovery of their liver function. As to animal experiments in this respect, rats with galactosamine-induced hepatic damage showed accumulation of antibiotics (carbenicillin, cefazolin) in tissues other than the liver.

3) Studies on drug interaction: Patients receiving surgical operation under systemic anaesthesia show much higher blood levels 
of cephaloridine compared with those after recovery. Rats anaesthetized with pentobarbital, too, show higher tissue levels of carbenicillin than normal ones. Diazepam administration yields a higher level of the same antibiotic in rat brains. These findings suggest the varied influences of drug interactions on the distribution of chemotherapeutics in the body, which may subsequently influence the incidence of their side effects.

Antibacterial chemotherapeutics are nowadays the most important drugs responsible for drug-induced disorders because of their vast use in medical practice. Although contemporary knowledge in this field may be helpful to some extent towards decreasing unfortunate results during drug therapy, further intensive investigations on the pathogenesis of each untoward reaction to each drug should be promoted in order to arrive at some solution to this vexing problem.

\title{
(4) Recent Advance in Research on $\beta$-Lactam and Aminoglycoside Antibiotics
}

\author{
Nobuo Tanaka, M D \\ Institute of Applied Microbiology, University of Tokyo, Tokyo
}

\section{$\beta$-Lactam Antibiotics}

Penicillin ( $\mathrm{PG}$ ) and cephalosporin (CES) group of antibiotics contains $\beta$-lactam ring in the molecule. It has been concluded from the studies on structure-activity relationship that the $\beta$-lactam ring is essential for the biological activity. The mechanism of action of the antibiotics has been extensively studied by a number of investigators. The antibiotics inhibit D-peptidyl transferase (D-transpeptidase, D-carboxypeptidase, and DD-endopeptidase) reactions, which form cross-link peptide bridge of cell wall peptidoglycan. D-peptidyl transferase may be the primary site of action or chemoreceptor of the antibiotics. The selective toxicity of the antibiotics is due to D-peptidyl transferase, which is contained in bacteria but not in mammals. A variety of $\beta$-lactam antibiotics has been obtained from microorganisms and/or semi-synthesized. They show a variety of biological activities, including those against resistant bacteria, fungi, and neoplasms. It is expected that the research on $\beta$-lactam antibiotics will be progressed in future and occupy more important position in the field of chemotherapy.

The studies on semi-synthetic penicillins provided the drugs effective against PCresistant Staphylococci, broad spectrum penicillins, and oral penicillins. Semi-synthetic cephalosporins were also developed. They show broad antimicrobial spectrum and are effective against PG-resistant organisms. The success in chemical transformation of penam ring (PG) to cephem ring (CES) contributed to the progress of semi-synthetic cephalosporins.

\section{New semi-synthetic penicillins}

New penicillins, in which a variety of carboxylic acid derivatives bound to the amino group in the side chain of ampicillin or amoxicillin, has been synthesized and found to be effective against Pseudomonas aeruginosa and other organisms.

In most of semi-synthetic penicillins, the 6-acyl group is modified, or 3-carboxyl group is esterified or substituted by tetrazolyl group. A new chemical method, modifying 2-methyl group, has been recently developed, 